ARTICLE

DOI: $10.1038 /$ s41467-018-05465-1

\title{
Visualization of translation termination intermediates trapped by the Apidaecin 137 peptide during RF3-mediated recycling of RF1
}

Michael Graf (10 1, Paul Huter ${ }^{1}$, Cristina Maracci (D) 2, Miroslav Peterek ${ }^{3}$,

Marina V. Rodnina (i) ${ }^{2} \&$ Daniel N. Wilson (D) ${ }^{1}$

During translation termination in bacteria, the release factors RF1 and RF2 are recycled from the ribosome by RF3. While high-resolution structures of the individual termination factors on the ribosome exist, direct structural insight into how RF3 mediates dissociation of the decoding RFs has been lacking. Here we have used the Apidaecin 137 peptide to trap RF1 together with RF3 on the ribosome and visualize an ensemble of termination intermediates using cryo-electron microscopy. Binding of RF3 to the ribosome induces small subunit (SSU) rotation and swivelling of the head, yielding intermediate states with shifted P-site tRNAs and RF1 conformations. RF3 does not directly eject RF1 from the ribosome, but rather induces full rotation of the SSU that indirectly dislodges RF1 from its binding site. SSU rotation is coupled to the accommodation of the GTPase domain of RF3 on the large subunit (LSU), thereby promoting GTP hydrolysis and dissociation of RF3 from the ribosome.

\footnotetext{
${ }^{1}$ Institute for Biochemistry and Molecular Biology, University of Hamburg, Martin-Luther-King-Platz 6, 20146 Hamburg, Germany. ${ }^{2}$ Department of Physical Biochemistry, Max Planck Institute for Biophysical Chemistry, Am Fassberg 11, Göttingen 37077, Germany. ${ }^{3}$ Central European Institute of Technology (CEITEC), Masaryk University, Kamenice 5, 62500 Brno, Czech Republic. Correspondence and requests for materials should be addressed to D.N.W. (email: daniel.wilson@chemie.uni-hamburg.de)
} 
T he termination phase of translation is signalled by the appearance of a stop codon of the mRNA within the A-site of the ribosome. In bacteria, stop codons are recognized by the decoding release factors RF1 and RF2, which facilitate release of the nascent polypeptide chain attached to the P-site tRNA ${ }^{1-4}$. RF1 and RF2 display distinct but overlapping stop codon specificities, such that RF1 decodes UAG and UAA and RF2 decodes UGA and UAA. Both RF1 and RF2 contain a universally conserved GGQ motif that is critical for peptide release ${ }^{5-9}$. Structures of RF1 and RF2 in complex with termination state ribosomes have revealed how conserved residues within the superdomain 2/ 4 specifically recognize the stop codon on the small subunit (SSU) 10-14. On the large subunit (LSU), the conserved GGQ motif within domain 3 is located at the peptidyltransferase center (PTC) and facilitates peptidyl-tRNA hydrolysis ${ }^{10-14}$. Following peptidyltRNA hydrolysis, the decoding RFs dissociate from the ribosome in a process that is stimulated by the action of a third release factor, the translational GTPase $\mathrm{RF}^{15,16}$.

Crystal structures of RF3 confirm structural similarity to other translational GTPases such as EF-Tu ${ }^{17,18}$. Like EF-Tu, RF3 binds to the ribosome with high affinity in the GTP form ${ }^{19-23}$. GTP hydrolysis is not required for the decoding factors to dissociate from the ribosome $\mathrm{e}^{21,22}$, but rather facilitates dissociation of RF3 from the ribosome ${ }^{22,23}$. RF3-GTP binds to ribosomes irrespective of the presence or absence of the decoding release factors, and also interacts with both pre- and post-hydrolysis complexes ${ }^{21-23}$. Biophysical studies indicate that binding of RF3 in the GTP form promotes the conversion of non-rotated RF1- or RF2-bound ribosomes into a rotated state ${ }^{22,24,25}$. Recently, an antimicrobial peptide that binds to the post-hydrolysis ribosome and prevents RF1 dissociation has been reported (Fig. 1a) ${ }^{22,26}$. This peptide, named apidaecin 137 (API), prevents RF1 dissociation even in the presence of $\mathrm{RF}^{22}$

Cryo-EM and X-ray structures exist of RF3-GDP(C/N)P (nonhydrolysable GTP analogues) bound to rotated ribosomes with P/ E-hybrid state tRNAs but without the decoding release factors (Fig. 1b) $17,27-29$. Although, the RF3 binding site overlaps with that of other translational GTPases, such as EF-Tu and EF-G, the G-domain of RF3 adopts a distinct orientation on the ribosome ${ }^{28,29}$. Superimposition of the RF3 and RF1/RF2 ribosome structures revealed no overlap in the factor binding sites, suggesting that RF3 indirectly promotes RF1/RF2 dissociation indirectly via inducing ribosomal subunit rotation ${ }^{14,17,27-30}$. A low-resolution ( $9.7 \AA$ A cryo-EM structure of RF1 and apo-RF3 (no nucleotide form) bound to a non-rotated ribosome has been determined ${ }^{30}$; however, the physiological relevance of this complex remains unclear ${ }^{21,23}$. By contrast, structures of decoding factors on termination state ribosomes in the
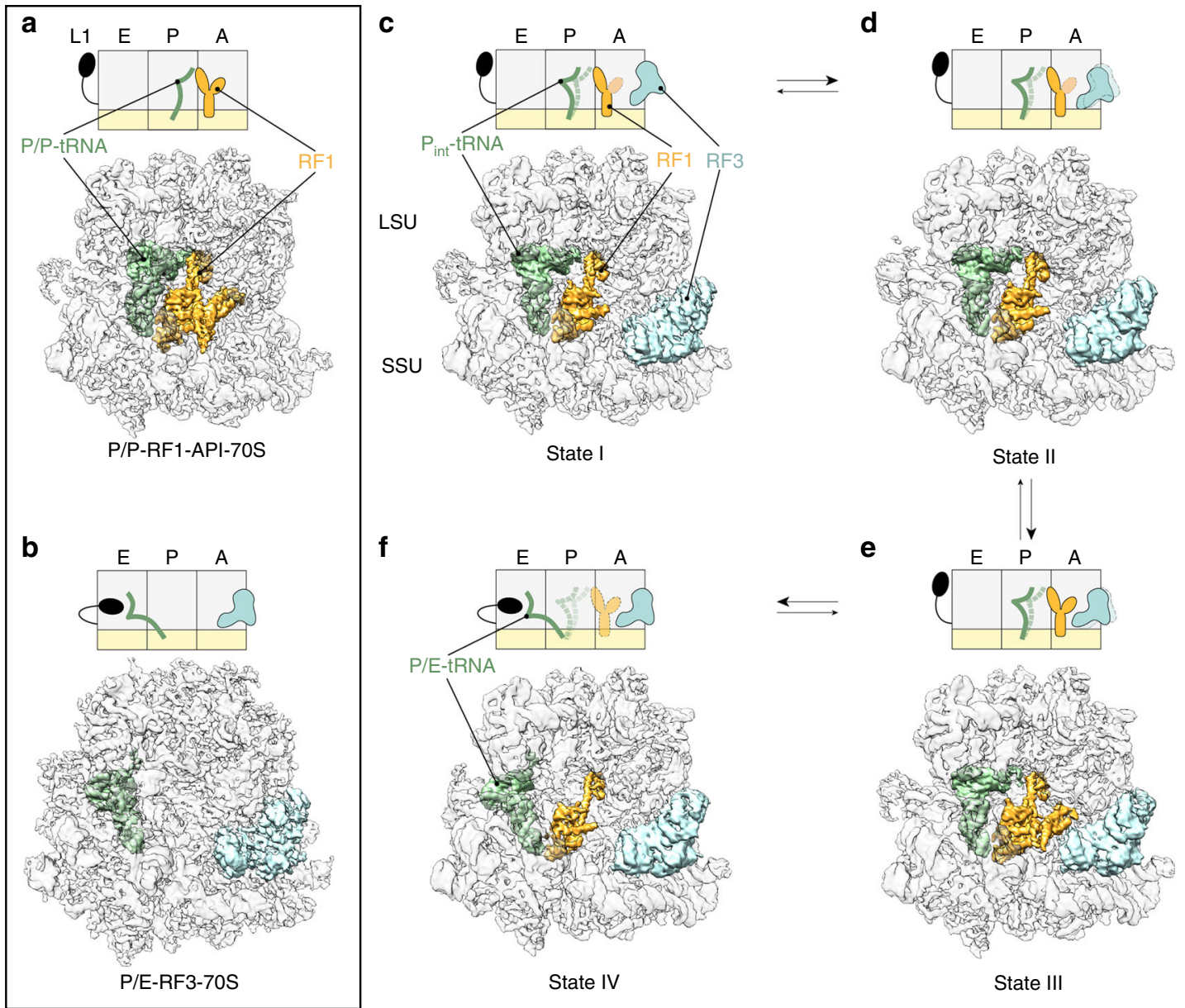

Fig. 1 Structures of RF1- and RF3-containing termination complexes. a-f Schematic representation (above) and electron density (below) for termination complexes containing a RF1 (orange) stalled by API on a non-rotated ribosome with a classical P/P-site tRNA (green) ${ }^{26}$, b RF3 (cyan) trapped by GDPCP on a rotated ribosome with hybrid P/E-site tRNA ${ }^{28}$, c-f state I-IV with RF1-GAQ (orange), RF3-GDPCP (cyan) bound to c-e partially rotated ribosomes with intermediate $\mathrm{P}$-site $\left(\mathrm{P}_{\text {int }}\right)$ tRNA (green) or $\mathbf{f}$ a fully rotated ribosome with a hybrid P/E-site tRNA (green). In the scheme, the SSU and LSU are coloured yellow and grey, respectively, with A-, P- and E-sites and the L1 stalk indicated, whereas flexible regions are indicated by increased transparency. In the map overviews, the electron density for the SSU and LSU has been filtered locally and is shown as a grey transparency so that the ligands can be easily seen within the ribosome 
presence of the active GTP-like form of RF3 have so far been lacking.

Here we present an ensemble of structures of tRNA, RF1 and RF3 trapped simultaneously on the ribosome using the termination-specific inhibitor API. The structures reveal that binding of RF3-GDPCP to the complex induces rotation of the SSU relative to the LSU. We do not observe interaction between RF1 and RF3 in any of the structures, suggesting that RF3 mediates dissociation of RF1 indirectly by inducing SSU rotation. SSU rotation also facilitates accommodation of RF3 on the LSU, where the $G$ domain interacts with the sarcin-ricin loop (SRL), which is necessary to stimulate GTP hydrolysis. Thus, RF3mediated subunit rotation plays a dual role during termination, namely, to dislodge the decoding release factors from the ribosome, but also to facilitate dissociation of RF3 itself.

\section{Results}

Cryo-EM structures of termination complexes with RF1 and RF3. In order to visualize both RF1 and RF3 simultaneously on the ribosome, we initially assembled a termination complex in vitro with RF1-GAQ mutant decoding a UAA stop codon in the A-site. This ribosome-tRNA-RF1 complex was then briefly incubated with RF3-GDPCP before being applied to cryo-grids and plunge-frozen. A low-resolution cryo-EM analysis revealed that the termination complex could be sorted into 8 classes, the majority of which contained either non-rotated ribosomes with RF1 but no RF3 or rotated ribosomes bearing RF3 but no RF1
(Supplementary Fig. 1a). The single class that appeared to contain both RF1 and RF3 had strong density for RF3 but poor density for RF1. Binding of RF1 and RF3 to the ribosome thus appeared to be nearly mutually exclusive, suggesting that RF3-GDPCP could recycle RF1-GAQ from the termination complex ribosomes, which is consistent with previous biochemical reports $22,24,25$. To increase the proportion of termination complexes containing both RF1 and RF3 bound simultaneously, we repeated the experiment in the presence of API, which was previously shown to prevent RF1 dissociation even in the presence of RF3-GTP ${ }^{26}$. Because API binds to the exit tunnel and replaces the nascent peptide, by addition of API we selectively stabilized those complexes where the nascent peptide was released despite the use of the RF1 mutant that is slow in catalysing hydrolysis of peptidyl-tRNA ${ }^{7,9}$. Using this complex, cryo-EM data was collected on a Titan Krios transmission electron microscope (TEM) with a Falcon II direct electron detector (DED) and processed with RELION2.1 ${ }^{31}$. A total of 525,595 ribosomal particles were sorted into eight distinct ribosomal subpopulations (Supplementary Fig. 1b). The four major subpopulations, states I-IV (15.2-22.4\%; 79,975-117,725 particles), all contained P-site tRNA, RF1 and RF3 but were conformational distinct from one another (Fig. 1c-f). States I-IV were refined to average resolutions of $3.8 \AA$ (State I, II) and $3.9 \AA$ (State III and IV) (Supplementary Fig. 1c-g and Table 1). Additionally, four minor subpopulations were present in the dataset, resulting in two additional low-resolution 70S-RF1-RF3 populations (see Methods), vacant $50 \mathrm{~S}$ subunits $(10.3 \%, 53,850$ particles) and RF3

Table 1 Cryo-EM data collection, refinement and validation statistics

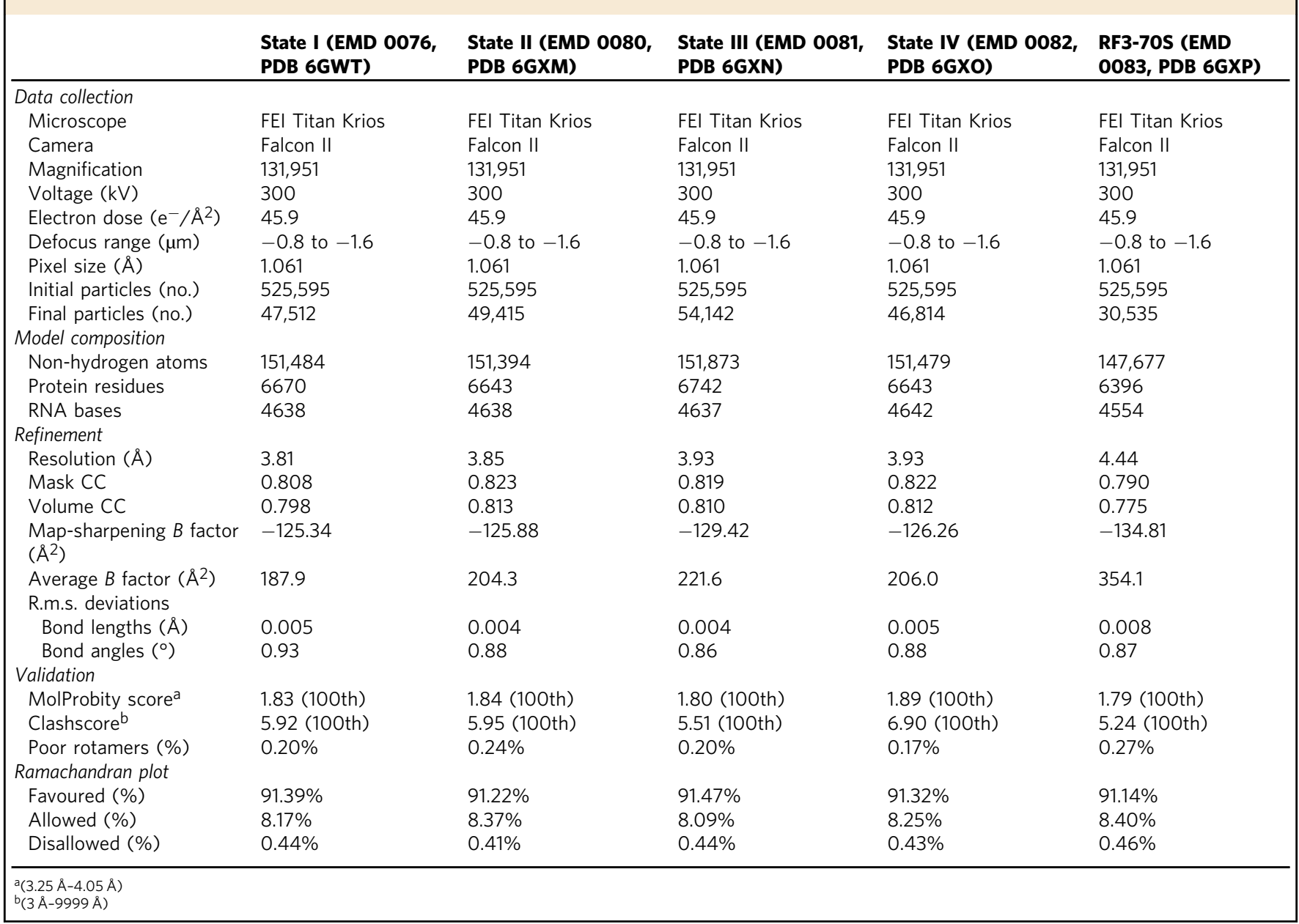


bound to rotated vacant 70 S ribosomes (5.8\%, 30,535 particles) (Supplementary Fig. 1b). Since the latter subpopulation did not contain a P/E-tRNA, we believe it represents a state where RF3-GDPCP bound directly to vacant 70S ribosomes, rather than to the ribosome-RF1-GAQ complexes. Local resolution calculations of the RF3-70S complex revealed that while the core of the ribosomal subunits reaches $4.0 \AA$ (Supplementary Fig. 2a, b), there is high conformational flexibility in this state. This is particularly evident in the rotation of SSU relative to the LSU and swivelling of the SSU head, but also in the positioning of the uL1 stalk and RF3 itself (Supplementary Fig. 2a, b). By contrast, states I-IV are more conformationally homogeneous, with local resolutions reaching $3.5 \AA$ within the core of both ribosomal subunits. Flexibility is predominantly observed at the periphery of the ribosome, namely, for the uL1, uL11 and bL12 stalks where local resolutions exceeded $7.5 \AA$ (Supplementary Fig. 2a, b). Local resolution calculations also indicated some conformational flexibility within the ribosome-bound ligands (Supplementary Fig. 2c). The resolution of the P-site tRNA and RF1 was highest (3.5-4.0 $\AA$ ) for the regions that interact with the SSU and LSU; whereas, the linking regions were significantly worse $(>7.5 \AA)$, such as the elbow of the tRNA, RF1 domain I and the linker between domains II and III of RF1 (Supplementary Fig. 2c). In states I-IV, the local resolution of the ligands was significantly better than that observed in the RF3-70S complex (Supplementary Fig. 2c). Molecular models of states I-IV and the RF3-70S complex were initially generated using rigid-body and domain-wise fitting of the ribosomal subunits, tRNA, RF1 and RF3 crystal structures to the cryo-EM map density, before manual adjustment, refinement and validation (Supplementary Fig. 2d; see Methods; Table 1). All states contained API bound within the ribosomal exit tunnel, where the interaction between Arg17 of API and the Gln235 (Q235 of the GGQ motif) of RF1 (Supplementary Fig. 3a-e) traps RF1 on the ribosome as reported previously ${ }^{26}$. a
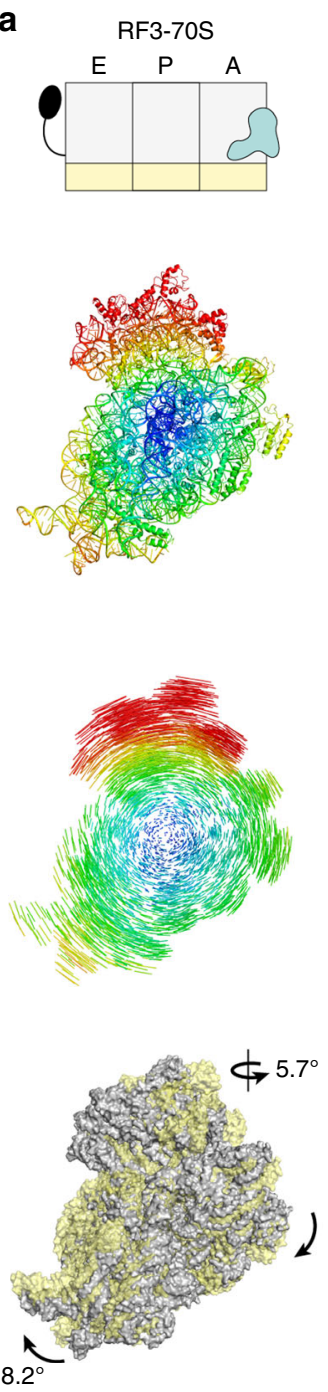

b
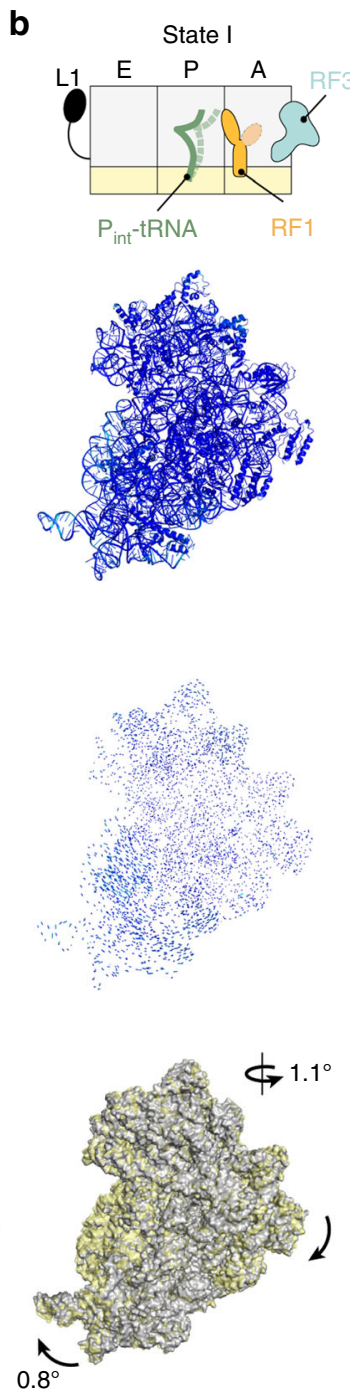

C
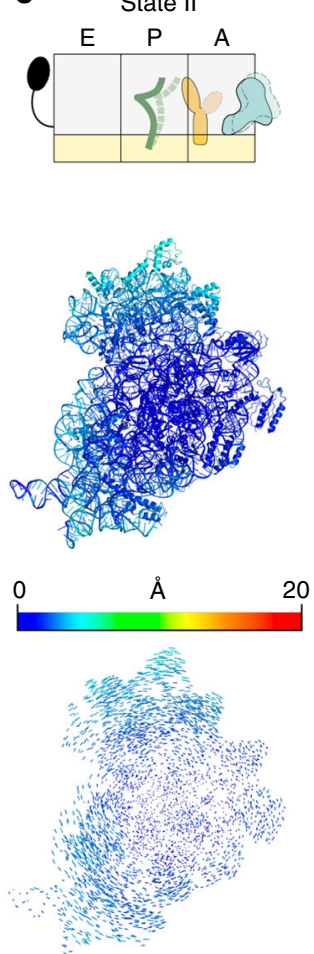

d
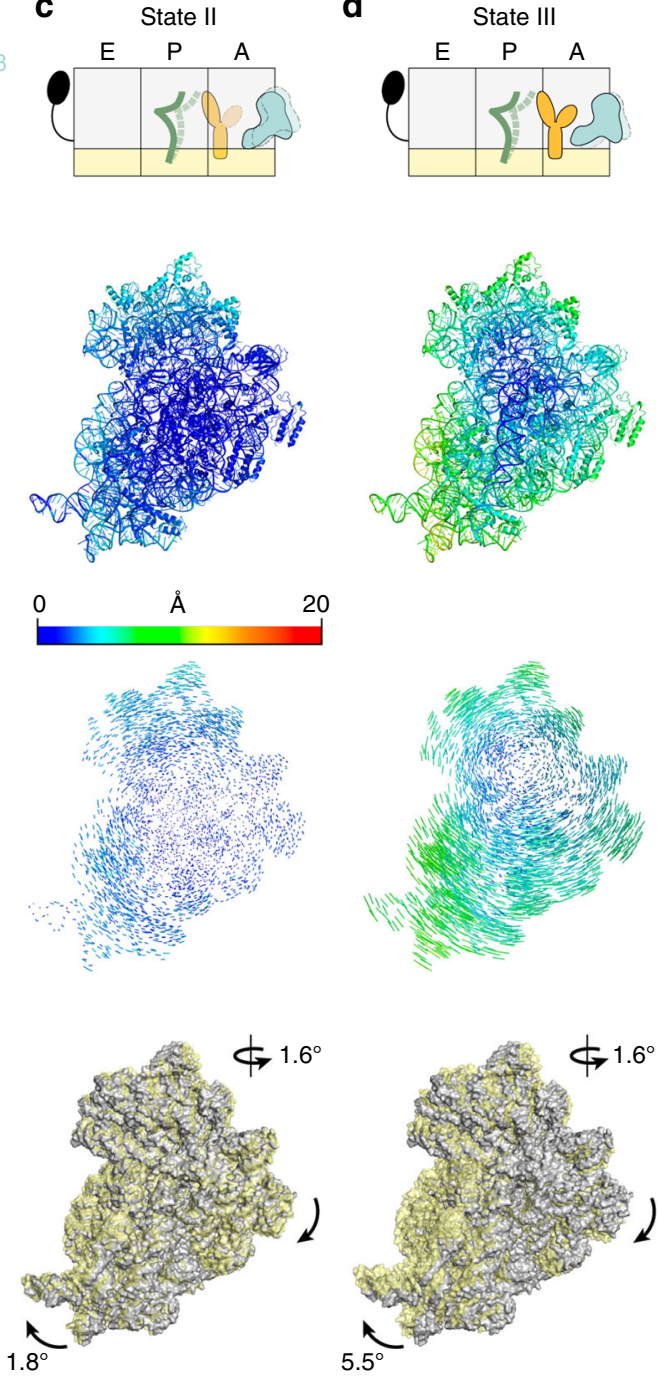

e
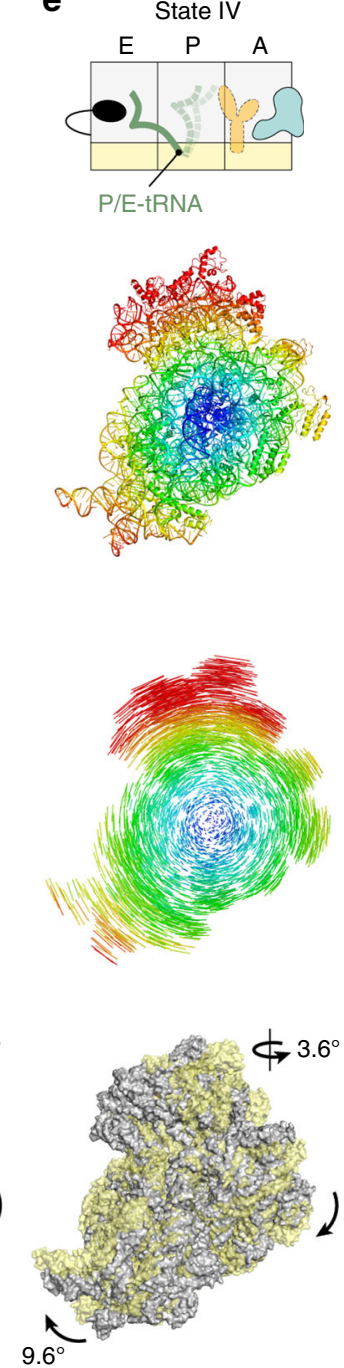

Fig. 2 Subunit rotation and head swivel observed in RF3-70S and termination state I-IV complexes. a-e Schematic representation (upper row) and SSU structures illustrating the degree of rotation relative to non-rotated RF1-API-70S reference structure ${ }^{26}$ as shown for a RF3-70S, b state I, c state II, d state III and e state IV. The distance each atom shifts relative to the reference structure is directly coloured on the SSU (second row), shown as coloured lines connecting the same atoms between the reference and the shifted structure (third row). Superimposition of cryo-EM maps (row four) of shifted SSU (yellow) relative to reference SSU (grey) based on LSU alignments, with degrees of SSU rotation (clockwise as viewed from intersubunit interface) and head swivel indicated 
RF3 induces ribosomal subunit rotation and head swivel. The major global movements distinguishing states I-IV and the RF3$70 S$ complex are the rotation of the SSU relative to the LSU as well as swivelling of the SSU head relative to the body (Fig. 2a-e and Supplementary Movie 1). The previously reported X-ray crystallography structures of RF3-70S complexes ${ }^{28,29}$ revealed SSU rotation of $\sim 10^{\circ}$ (clockwise when viewing the intersubunit interface of the SSU) compared to a classical (non-rotated) ribosome, such as the RF1-API-70S complex ${ }^{26}$ (Supplementary Table 1). We also observed a similar degree of subunit rotation in the RF3-70S complex (Fig. 2a), whereas states I, II, III and IV displayed a range of intermediate levels of rotation, namely, $0.8^{\circ}$, $1.8^{\circ}, 5.5^{\circ}$ and $9.6^{\circ}$, respectively (Fig. 2b-e and Supplementary Movie 1). In the X-ray crystallography structures of the RF3-70S complexes, the degree of head swivel differed dramatically from one another and was suggested to be dependent on the presence or absence of the hybrid P/E-site tRNA ${ }^{28,29}$. In the absence of the $\mathrm{P} / \mathrm{E}$-site tRNA, the head was swivelled $\sim 14^{\circ}$ compared to the body ${ }^{29}$, whereas only $\sim 3-4^{\circ}$ head swivelling was observed when the P/E-site tRNA was present ${ }^{28}$ (Supplementary Table 1 and Supplementary Movie 2). In our RF3-70S complex lacking a tRNA (Supplementary Fig. 2), we observed an intermediate level $\left(\sim 6^{\circ}\right)$ of head swivel (Fig. 2a, Supplementary Table 1 and Supplementary Movie 2). However, as noted above, the head is highly dynamic in our RF3-70S complex (Supplementary Fig. 2a, b) and thus the value reflects an average of multiple different swivel conformations of the head. This supports the suggestion that the large degree of head swivel observed in one of the X-ray crystallography structure of the RF3-70S complex is indeed due to the absence of the P/E-site tRNA ${ }^{29}$. Consistently, in states I-IV that contain $\mathrm{P}$ - or P/E-site tRNAs, the maximum head swivel observed was $\sim 3.6^{\circ}$ (Fig. $2 \mathrm{~b}-\mathrm{e}$ and Supplementary Table 1 ). Moreover, the degree of head swivel appeared to be loosely correlated to that of subunit rotation, as the degree of swivelling also increased from state I to IV, namely, $1.1^{\circ}$ to $3.6^{\circ}$, respectively (Fig. $2 \mathrm{~b}-\mathrm{e}$ ). The degree of rotation $\left(\sim 10^{\circ}\right)$ and head swivel $\left(\sim 4-6^{\circ}\right)$ observed here in state IV and RF3-70S complex is similar to that observed previously for translation elongation states with hybrid $\mathrm{A} / \mathrm{P}$ - and $\mathrm{P} / \mathrm{E}$-site tRNAs (Supplementary Table 1) ) $^{32-37}$.

Remodelled interactions between the P-site tRNA and P-loop. The SSU rotation and head swivel observed in states I-IV is also accompanied by a corresponding shift of the P-site tRNA (Fig. 3a and Supplementary Movie 1). Compared to the classical P-site tRNA in the RF1-API-70S complex in the absence of RF3, the tRNA is rotated towards the E-site by $\sim 13^{\circ}$ in state I-III and by $\sim 34^{\circ}$ in state IV (Fig. 3a). For state IV, this generates a hybrid
P/E-tRNA where the CCA-end of the tRNA interacts with the Esite on the LSU, as observed for hybrid P/E-site tRNA during translation elongation ${ }^{32-37}$. By contrast, the intermediate P-site tRNA positions observed in states I-III still have the CCA-end located in the vicinity of the peptidyltransferase center (PTC) of the LSU. A classical P-site tRNA is positioned at the PTC via base-pairing of the C74 and C75 of the CCA-end of the P-site tRNA with nucleotides G2252 and G2251, respectively, of the P-loop (helix H80) of the 23S rRNA (Fig. 3b). By contrast, the rotation of the P-site tRNA observed in states I-III results in a shift of the CCA-end of the P-site tRNA out of the PTC by $\sim 9 \AA$ (Fig. 3c, d). Surprisingly, we observed a register shift in the basepairing of the CCA-end of the P-site tRNA intermediate with the P-loop nucleotides, such that C74 and C75 were base-paired with G2253 and G2252, respectively. In addition, A73 of the P-site tRNA appeared to flip to establish a non-canonical wobble basepair with C2254 (Fig. 3c, d). In contrast to the canonical P-site tRNA where the electron density was clearly resolved for the complete CCA-end, no density was visible for the terminal A76 of the P-site tRNA intermediate in states I-III, therefore assignment of the acylation state of the $\mathrm{P}_{\text {int }}$-tRNA was not possible (Supplementary Fig. $3 \mathrm{f}-\mathrm{h}$ ). We do not believe that the register shift of the CCA-end of the $\mathrm{P}_{\text {int }}$-tRNA is by two nucleotides, such that A76, C75 and C74 base-pair with G2252, G2253 and C2254, respectively, because the density does not support a purine-purine (A76-G2252) interaction in the first position, nor a pyrimidinepyrimidine (C74-C2254) interaction at the third position (Supplementary Fig. 3i). Moreover, the density for A73 observed in the P/P-tRNA is not observed in the $\mathrm{P}_{\text {int }}$-tRNA (Supplementary Fig. 3j-1), which would be expected if C74 interacts with C2254 and prevents A73 from establishing this interaction. Regardless of one or two nucleotide register shift, to our knowledge, such rebase-pairing of the CCA-end of a P-site tRNA with the P-loop has not been observed previously.

Subunit rotation accommodates RF3 on the LSU. In the RF3$70 \mathrm{~S}$ complex and states I-IV, RF3 is observed to rotate as a rigid body together with the SSU (Fig. 4a and Supplementary Movie 1). The overall conformation of RF3 is the same as observed in the previous RF3-70S complexes ${ }^{28,29}$, differing from the free RF3 form by the shift of domains 2 and 3 relative to domain $1^{17,18,28,29}$. Because of the coordinated movement of RF3 with the SSU, the interactions between domain 2 and 3 of RF3 with ribosomal protein uS12 and helices h5 and h15 of the $16 \mathrm{~S}$ rRNA, as described previously ${ }^{28,29}$, are maintained in all states. Relative to the LSU, however, RF3 moves by up to $10-11 \AA$ when comparing states I-IV, bringing the G-domain of RF3 in state IV into
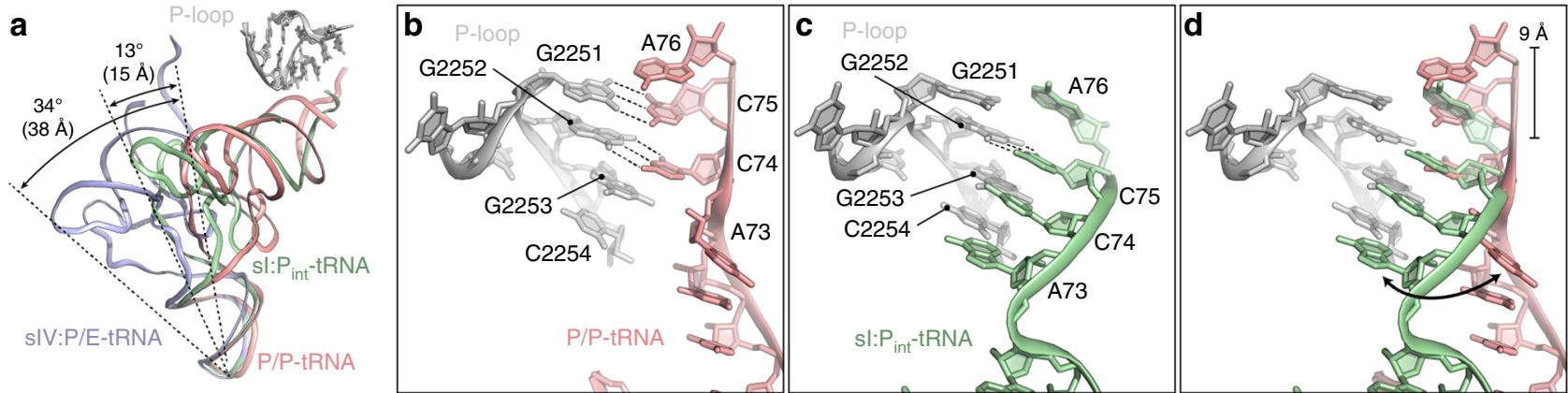

Fig. 3 Small subunit rotation induces P-site tRNA rotation. a Comparison of the relative position of a classical P/P-tRNA (salmon) from the RF1-API-70S complex ${ }^{26}$ to $P_{\text {int }}$ tRNA (green) conformation observed in state I and the hybrid P/E-site tRNA observed in state IV (slate), with the degree of rotation and distance shifted indicated. The P-loop of the $23 \mathrm{~S}$ rRNA is shown for reference. $\mathbf{b}$ The P/P-site tRNA (salmon) in the RF1-API-70S complex ${ }^{26}$ and $\mathbf{c}$ the $\mathrm{P}_{\text {int }}{ }^{-}$ tRNA (green) in state I interact with nucleotides within the P-loop ( $\mathrm{H} 80$ ) of the $23 \mathrm{~S}$ rRNA. Potential hydrogen bonds are indicated with dashed lines. d Superimposition of $\mathbf{b}$ and $\mathbf{c}$ with arrow indicating the flipped A73 nucleotide 
closer proximity of the sarcin-ricin loop (SRL, H95 of the 23S rRNA) (Fig. 4b and Supplementary Fig. 4a). Because the SRL is critical for stimulating the GTPase activity of translation factors $^{38}$, this suggests that the SSU rotation is necessary for efficient activation of the GTPase activity of RF3. Evidence for progressive accommodation of translational GTPases on the LSU, as well as GTPase activation by the SRL, has been observed for other translational GTPases, such as IF2 ${ }^{39,40}, \mathrm{eEF}^{\mathrm{A}} \mathrm{A}^{41,42}$, SelB ${ }^{32}$ and $\mathrm{EF}-\mathrm{Tu}^{43}$ (Supplementary Fig. 4b). However, in these latter cases, the translational GTPases bind to non-rotated ribosomes and accommodation of the GTPase appears to be mediated by SSU domain closure ${ }^{32,41-43}$, rather than by rotation as observed here for RF3. Despite the sequence and structural conservation of the G-domain of RF3 with other translation GTPases, the G-domain of RF3 adopts a distinct orientation on the ribosome ${ }^{28,29}$. With respect to the SRL, the G-domain of RF3 is rotated by $24-31^{\circ}$ when compared with other translational GTPases, such as IF2, EF-Tu and EF-G (Supplementary Fig. 4c-f). Within the limits of the resolution, the switch II loop conformation of the G-domain of RF3 in states I-IV is consistent with that observed in previous structures of $\mathrm{RF}^{17,18,28,29}$, where it interacts with the $\gamma$-phosphate of the GDPCP (Fig. 4c). While the switch I loop is poorly ordered in states II-IV, we observed a well-defined conformation in state I, where it interacts with ribosomal proteins uL14 and bL19, but not with the SRL (Fig. 4c). The switch I loop is disordered in many previous structures of RF3 ${ }^{17,18,28}$, although ordered conformations were previously reported in the E. coli RF3-70S structure ${ }^{29}$ as well as the Desulfovibrio vulgaris RF3 in complex with the alarmone $\mathrm{ppGpp}^{18}$. However, they are significantly different from that observed here in state I
(Supplementary Fig. 5a-f). The interaction of the switch I loop conformation of RF3 with uL14 and bL19 is the only direct contact that RF3 establishes with the LSU in state I, and thus may be important for facilitating accommodation of RF3 on the ribosome.

A dual role for bL12 during translation termination. The GTPase activity of translational GTPases, such as IF2 ${ }^{44,45}$, EF-Tu and EF-G $\mathrm{G}^{46,47}$ as well as RF3 ${ }^{44}$, is stimulated by the ribosomal bL12 stalk, a pentameric complex consisting of four copies of bL12 tethered to the ribosomal protein uL10. In states I-IV, we observe an additional density that we attribute to the C-terminal domain (CTD) of one copy of bL12 interacting with the G' domain of RF3 (Fig. 5a, b), as observed in previous RF3-70S cryoEM structures 17,30 , and consistent with $\mathrm{NMR}^{48}$ and mutagenesis studies $^{44}$. bL12 was shown to stimulate Pi release from EF-G following hydrolysis of GTP to GDP and Pi, enabling the low affinity GDP conformation of EF-G to be adopted and thereby facilitating the dissociation of EF-G from the ribosome ${ }^{49}$. However, bL12 has also been implicated in promoting binding of translational GTPases, such as EF-G and EF-Tu, to the ribosome ${ }^{47,50}$. Surprisingly, in state III, an extra density is observed that we attributed to a second CTD of bL12, which bridges domain I of RF1 with the ribosomal protein uL11 (Fig. 5b). This extra density can also be seen in the cryo-EM map of the previously reported RF1-API-70S complex (Supplementary Fig. $5 g, h)^{26}$, but cannot be seen in states I-II and IV due to the delocalized RF1 domain I. In state III, domain I of RF1 is better resolved, compared to states I and II, due to head swivelling on

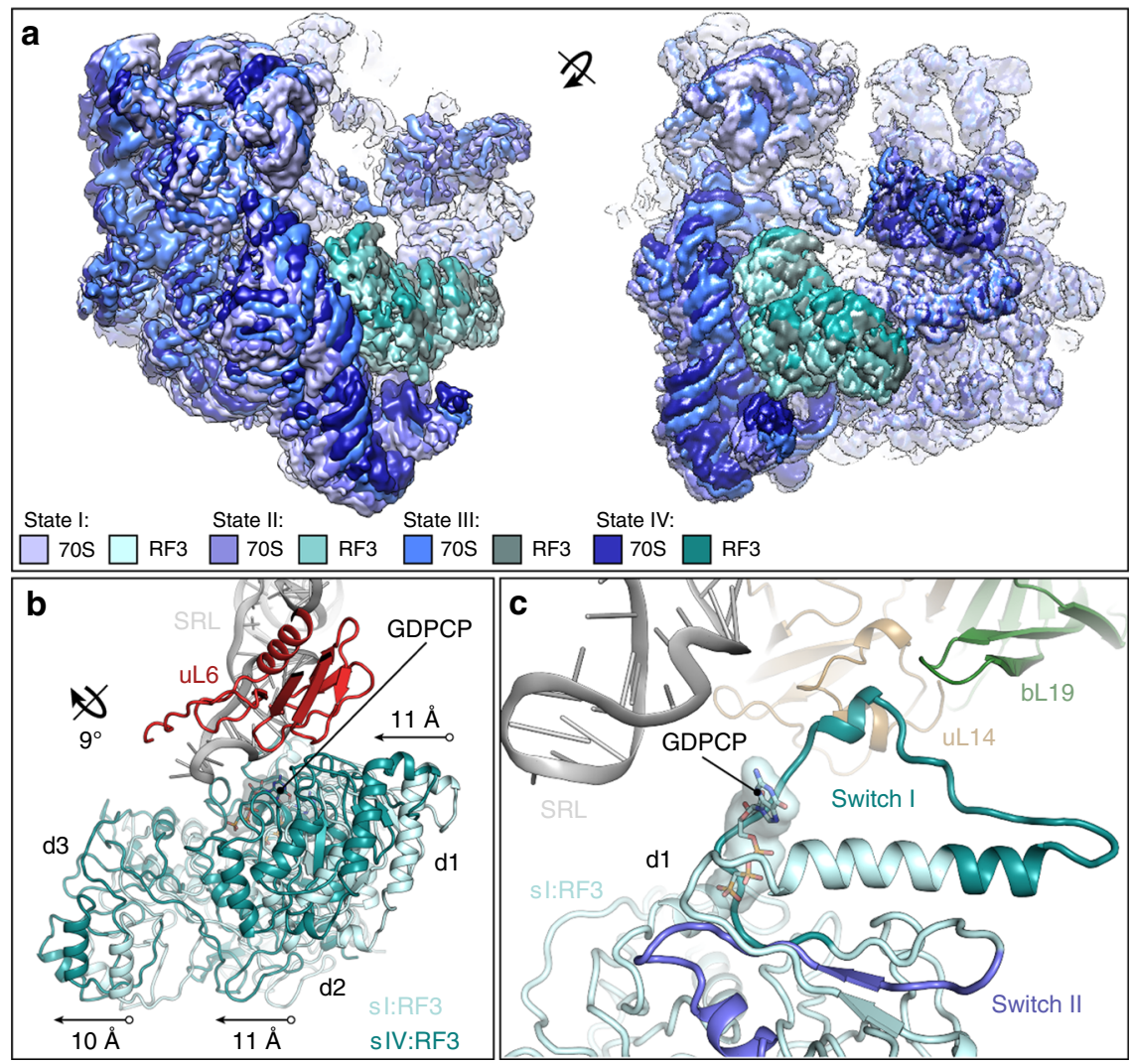

Fig. 4 Small subunit rotation leads to RF3 accommodation on the large subunit. a Two views of the cryo-EM maps of the $70 \mathrm{~S}$ ribosome (different shades of blue) and RF3 (different shades of green) from states I-IV, illustrating the coupled rotation of the SSU and RF3 relative to the LSU. $\mathbf{b}$ Comparison of the binding site of RF3 in state I (pale cyan, sl:RF3) and state IV (teal, sIV:RF3) relative to ribosomal protein uL6 (red) and sarcin-ricin loop (SRL, grey). The distance shifted of each domain (d1-d3) of RF3 between states I and IV is indicated. c View showing the G domain of RF3 in state I (pale cyan, sl:RF3) with switch I (teal), switch II (slate), GDPCP and proximity to SRL (grey), ribosomal proteins uL14 (tan) and bL19 (green) 


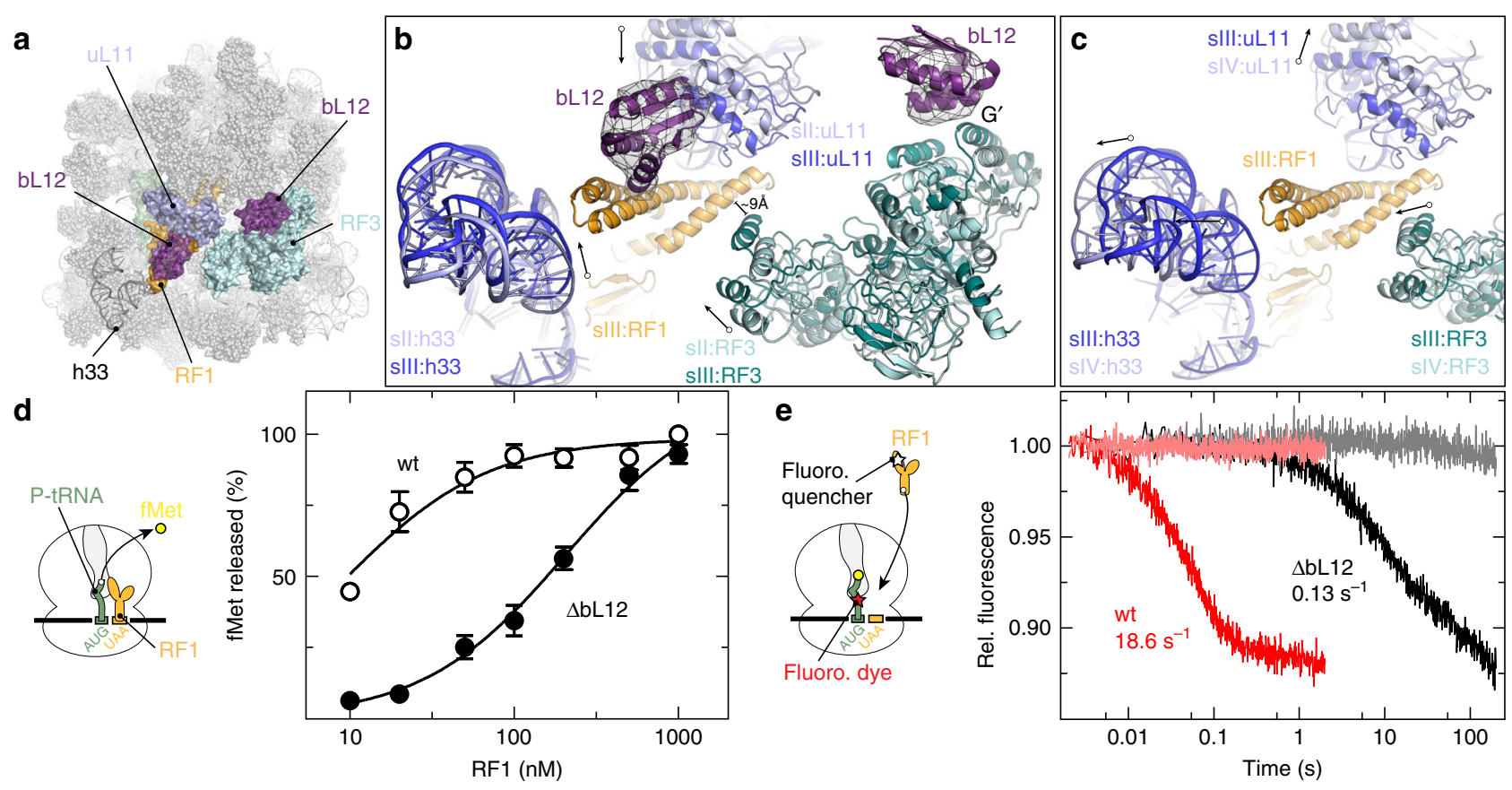

Fig. 5 Interaction of bL12 with RF1 and RF3 in state III. a Overview showing the relative position of RF1 (orange), RF3 (pale cyan), uL11 (light blue), $23 \mathrm{~S}$ rRNA helix h33 (dark grey) and two copies of the bL12 CTD (purple) on the ribosome (light grey). b Comparison of state II position for RF3 (pale cyan), uL11 and h33 (light blue) with state III positions for RF3 (teal), uL11 and helix h33 (dark blue). In state III, RF1 domain I (orange) becomes ordered and density (grey mesh, filtered to $7 \AA$ ) for two copies of bL12 CTD (purple) are observed. c Comparison of state III positions from (b) with state IV positions for RF3 (pale cyan), uL11 and h33 (light blue). d Peptide hydrolysis by RF1 in the presence of wild type (wt, open circles) or bL12-depleted ( $\Delta$ bL12, closed circles) ribosomes. Pre-hydrolysis (PreHC) complexes $(0.01 \mu \mathrm{M})$ were incubated with increasing concentrations of RF1 for $10 \mathrm{~s}$ at $37^{\circ} \mathrm{C}$. Solid lines represent the hyperbolic fit of the experimental points. Error bars represent the standard deviation of the mean for four technical replicates from two independent biological experiments. The apparent affinities of RF1 for wt and $\Delta b L 12$ PreHCs are $9 \pm 1$ and $210 \pm 30 \mathrm{nM}$, respectively. e Time courses of RF1-GAQ $\mathrm{Qsy}_{9}$ $(0.3 \mu \mathrm{M})$ binding to PreHC $\mathrm{Flu}_{\mathrm{u}}(0.05 \mu \mathrm{M})$ prepared with wt (red) or $\Delta \mathrm{bL12}$ (black) ribosomes. Buffer controls are shown in salmon and grey, respectively. Traces shown are the average of four to five technical replicates

the SSU and closure of the uL11 stalk base of the LSU (Fig. 5b). By contrast, transition from state III to IV involves additional head swivelling and opening of the uL11 stalk base, which leads to loss of interaction and destabilization of domain I of RF1 (Fig. 5c). Thus, our observations suggest that in addition to stimulating the GTPase activity of RF3, bL12 may also be involved in facilitating the binding of the termination decoding factors to the ribosome. To test this hypothesis we prepared ribosome termination complexes using bL12-depleted $(\Delta \mathrm{bL} 12)$ ribosomes $^{46}$ and determined the apparent affinity of RF1 by peptide hydrolysis (Fig. $5 \mathrm{~d}$, see Methods). The $\Delta \mathrm{bL} 12$ ribosomes showed a markedly decreased affinity for RF1, compared to wild-type (wt) ribosomes bearing bL12 (Fig. 5d). We further confirmed this result by measuring the kinetics of RF1 binding via fluorescence resonance energy transfer from a dye-labelled fMet-tRNA ${ }^{\mathrm{fMet}}$ and a quencher-labelled RF1-GAQ (RF1-GAQ ${ }_{Q \text { sy9 }}$ ) (Fig. 5e ${ }^{26}$. Binding of RF1-GAQ to the $\triangle \mathrm{bL} 12$ ribosomes was 150 -fold slower than to wt ribosomes, indicating that the contribution of bL12 to RF1 recruitment is large.

Subunit rotation destabilizes RF1 binding. Unlike the previous low-resolution (9.7 $\AA$ ) cryo-EM structure of apo-RF3-RF1-70S complex where contact was reported between RF1 and RF3 ${ }^{30}$, we do not observe interaction between RF1 and RF3 in any of the structures determined here. The closest distance between the two factors is seen in state III where domain I of RF1 becomes ordered such that $\alpha$-helix 2 of domain 1 of RF1 comes within $9 \AA$ of RF3 domain 3 (Fig. 5b). Moreover, since RF3 can recycle RF1 variants lacking domain $\mathrm{I}^{51}$, we conclude that RF3 does not use a direct steric overlap in binding site to dissociate RF1 from the ribosome. Instead, our results suggest that RF3 dislodges $\mathrm{RF} 1$ from its binding site, indirectly, by inducing SSU rotation, as postulated previously ${ }^{17,28,29}$. The density for RF1 remains well-resolved through-out states I-III, indicating that RF1 is stably bound to the ribosome despite the increase in rotation $\left(0.8-5.5^{\circ}\right)$ of the SSU (Fig. 6a). By contrast, the density for RF1 is poorly resolved in state IV, indicating that RF1 becomes destabilized from its binding site on the ribosome (Fig. 6a, b). Increased flexibility of RF1 in state IV is also supported by local resolution calculations (Supplementary Fig. 2c). Transition from state III to IV involves further clockwise rotation of the SSU (from 5.5 to $9.6^{\circ}$ ), which results in a shift of domain $2 / 4$ of RF1 by $4 \AA$ compared to state III (Fig. 6a, c). Because domain 3 of RF1 remains static at the PTC of the LSU, the movement can be described by a rotation of $6^{\circ}$ that is accommodated by the long flexible linkers connecting domain $2 / 4$ with domain 3 of RF1 (Fig. 6c). We believe that it was possible to capture the RF1 conformation in state IV only because the complex was formed in the presence of API, which prevented the complete dissociation of RF1 from the rotated ribosome. We note that transition from state III to IV also encompasses additional head swivel (from $1.6^{\circ}$ to $3.6^{\circ}$ ) as well as opening of the bL12 stalk base (Fig. 5c), both of which destabilize domain 1 of RF1 and may facilitate dissociation of RF1 from the ribosome. Additionally, the formation of a hybrid P/E-tRNA due to the fully rotated SSU in state IV also leads to loss of interaction of RF1 with the P/P-tRNA, which may also contribute to destabilization of RF1 binding. Specifically, the interactions between Glu155 and His156 in domain 2/4 and Arg261 in domain 3 of RF1 with the ASL and CCA-end of a P/ P-tRNA, respectively, are lost upon P/E-tRNA in state IV (Fig. 6d, e). 


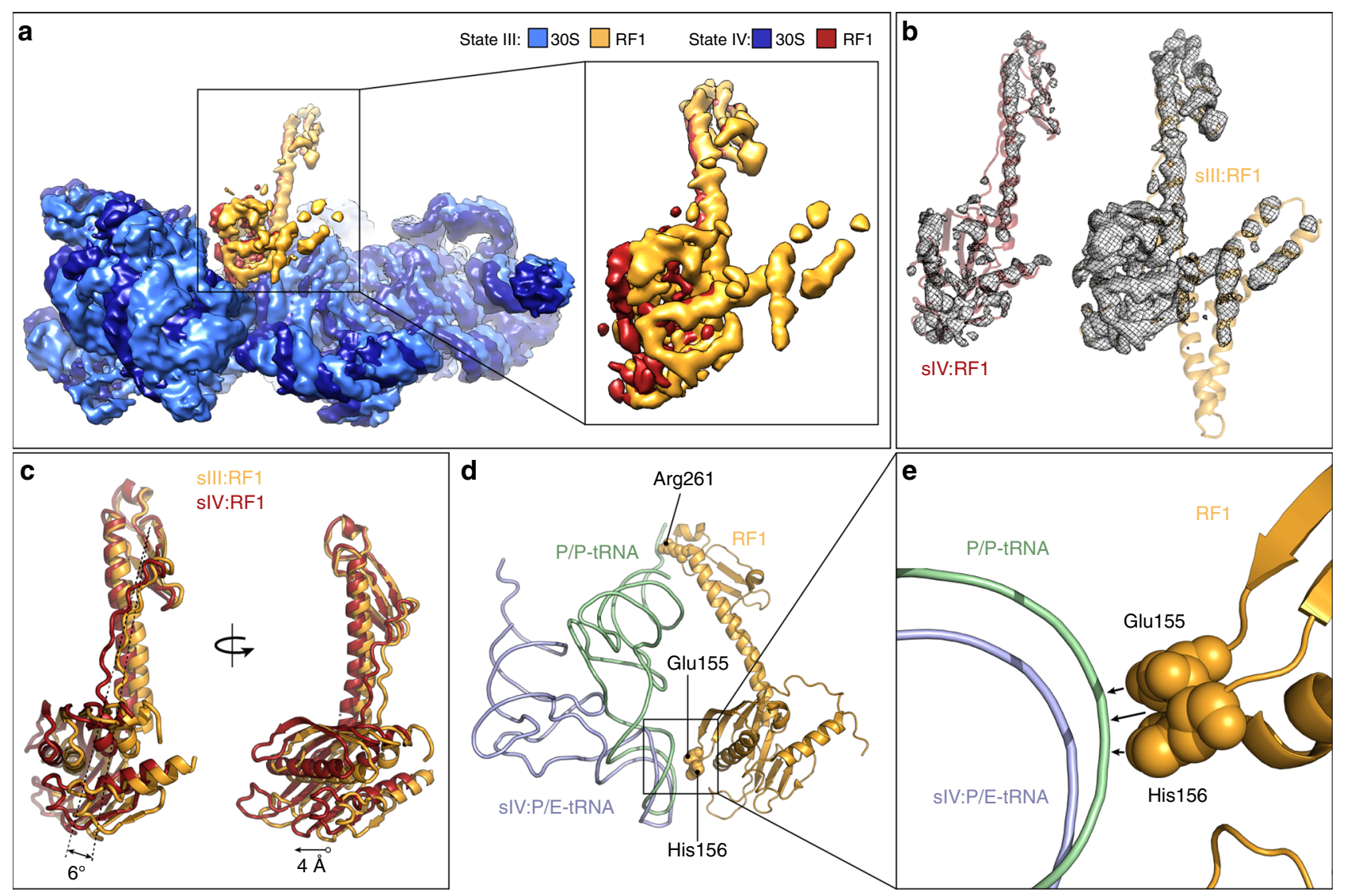

Fig. 6 RF3-induced subunit rotation destabilizes RF1 binding. a Cryo-EM map of SSU (light blue) and RF1 (orange) from state III compared with SSU (dark blue) and RF1 (red) from state IV. b Isolated cryo-EM electron densities (grey mesh) with molecular models for RF1 from state III (orange) and state IV (red) shown at the same contour level based on comparison with the SSU density. c Domain 2/4 of RF1 from state III (sIII:RF1, orange) is rotated by $6^{\circ}$ and shifted by $4 \AA$ compared to RF1 from state IV (sIV:RF1, red). d, e Contacts (arrowed) between RF1 (orange) and P/P-tRNA (green) are lost upon formation of the hybrid P/E-tRNA (light blue). Amino acids of RF1 that contact P/P-tRNA are shown as spheres. $\mathbf{e}$ Zoom of $\mathbf{d}$ showing the presence or absence of RF1 contacts with the ASL of $\mathrm{P} / \mathrm{P}$ - or $\mathrm{P} / \mathrm{E}-\mathrm{tRNA}$, respectively

a

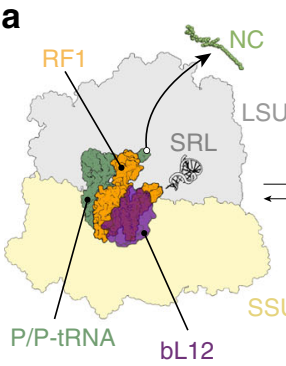

b

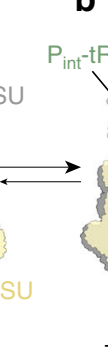

(1)

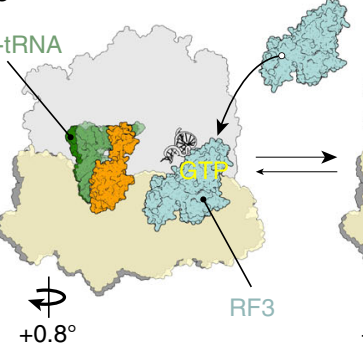

C

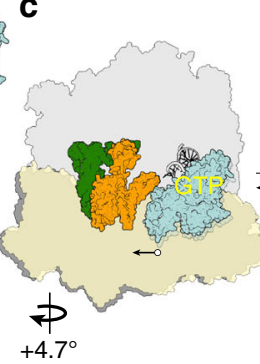

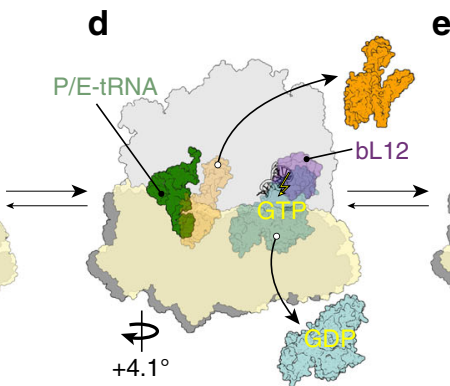

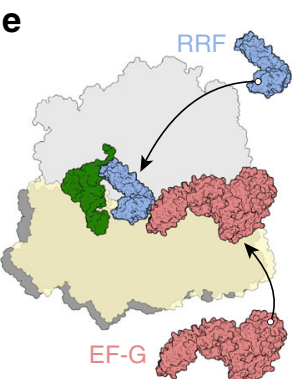

Fig. 7 Model for RF3-mediated dissociation of RF1 from the ribosome. a Binding of decoding release factors, such as RF1 (orange) to the non-rotated termination state ribosome, leads to release of the nascent polypeptide chain (NC) from the P-site tRNA (green). Binding of RF1 to the ribosome is facilitated by interaction of bL12 CTD (purple) with domain 1 of RF1. b RF3 (cyan) in the GTP conformation binds to the ribosome-RF1 complex. RF3 binding induces a slight rotation of the SSU that promotes formation of the partially rotated P-site tRNA conformation ( $P_{\text {int }}$-tRNA). c Additional SSU rotation and head swivel stabilizes domain I of RF1 and induces a closed conformation of uL11 (Fig. 5b, c). d Further SSU rotation leads to destabilization of RF1, promoting its dissociation from the ribosome, as well as accommodation of RF3 on the LSU in close proximity to the sarcin-ricin loop (SRL), thus facilitating hydrolysis of GTP to GDP and dissociation of RF3-GDP from the ribosome. e The rotated ribosome with a P/E-tRNA is recognized by the ribosome recycling factor (RRF) and $\mathrm{EF}-\mathrm{G}$, which recycle the post-termination complex for the next round of translation

\section{Discussion}

Based on our ensemble of termination intermediates (states I-IV), as well as the available literature, we suggest a revised model for RF3-mediated dissociation of RF1 during translation termination (Fig. 7a-e). A stop codon in the A site is recognized by the decoding factors RF1 (or RF2), which catalyse hydrolysis of the peptidyl-tRNA (Fig. 7a). The observed interaction between the CTD of one copy of bL12 and domain 1 of RF1 in the RF1API-70S complex (Fig. 7a), as well as the RF1-API-RF3-70S complex (state I), is supported by our experimental findings 
(Fig. 5d, e) showing that bL12 plays an important role in delivery of RF1 to the ribosome, as reported previously for EF-Tu and EF$\mathrm{G}^{46,47}$. To date, all reported structures of decoding factors RF1 (and RF2) were bound to ribosomes with non-rotated conformations ${ }^{14,52,53}$, including the apo-RF3-RF1-70S complex $^{30}$. By contrast, we observe that binding of RF3 to the ribosome in states I-IV that contain RF1 induces rotation of the SSU relative to the LSU (Fig. 7b-e). While we have ordered states I-IV based on the degree of subunit rotation, we acknowledge that the exact biological sequence of states cannot be ascertained from our study. Nevertheless, we believe that this order produces a logical sequence of events that provide a working model for decoding factor recycling by RF3. In state I, we observe that even a small degree $\left(0.8^{\circ}\right)$ of subunit rotation induced by RF3 results in a shifted position of the P-site tRNA, such that it partially rotates out of the PTC in the direction of the E-site (Fig. 7b). The resulting intermediate $\mathrm{P}$-site tRNA ( $\mathrm{P}_{\text {int }}$-tRNA) displays register shift in the base-pairing between the CCA-end of the tRNA and the P-loop of the PTC (Fig. 3). Moreover, we observe ordering of the switch I loop in the G domain of RF3 (Fig. 4c), which establishes the sole interaction with the LSU and may therefore be important for accommodation of RF3 on the ribosome. Further subunit rotation $\left(+4.7^{\circ}\right)$ as well as the head swivel observed in state III leads to a stabilization of domain 1 of RF1 and further accommodation of RF3 on the LSU (Fig. 7c). In state III, domain I of RF1 comes within $9 \AA$ of RF3 (Fig. 5b), which is the closest distance between the two factors in any of the structures reported here. The absence of an observed interaction between RF1 and RF3 in states I-IV suggests that RF3 must indirectly induce RF1 dissociation from the ribosome. Indeed, in state IV, we observe that further rotation $\left(+4.1^{\circ}\right)$ of the SSU leads to a destabilization in the binding of RF1 (Fig. 7d). We note that transition from states III to IV also encompasses additional head swivelling and movement of the uL11 away from RF1, as well as loss of interaction with the P/E-tRNA, which may also contribute to the destabilization of RF1 binding (Fig. 5c). The large degree $\left(9.6^{\circ}\right)$ of SSU rotation observed in state IV brings the G domain of RF3 into close proximity of the SRL on the LSU (Fig. 7d). Thus, we predict that subunit rotation is necessary to stimulate the GTPase activity of RF3 and thereby facilitate dissociation of the low affinity RF3-GDP from the ribosome (Fig. 7d). As reported previously, we also observe interaction of the CTD of bL12 with the G' domain of RF3 in states I-IV, suggesting that bL12 may also play a role in GTPase activation and dissociation of RF3 (Fig. 7d). Because RF1 and RF3 are trapped on the ribosome with API and GDPCP in our structures, we cannot distinguish the order of dissociation of RF1 and RF3. Nevertheless, our structures suggest that RF1 and RF3 dissociation are both coupled to full rotation of the SSU, and are therefore likely to occur within a very similar timeframe, as reported recently using biophysical assays ${ }^{22,54}$. We note the product remaining after the action of RF1 and RF3 is a rotated ribosome complex with a hybrid P/E-site tRNA, which is the exact substrate for the next phase of translation, namely, ribosome recycling via the binding of RRF and EF- ${ }^{55-60}$ (Fig. 7e).

\footnotetext{
Methods

Preparation of the ribosomal complex. Ribosomes from the E. coli strain MRE600, initiation factors, $\mathrm{fMet}_{\mathrm{tRNA}} \mathrm{fMet}^{\mathrm{fm}}$ an its fluorescein-labelled derivative were prepared as described ${ }^{61,62}$. bL12-depleted ribosomes were prepared by $\mathrm{NH}_{4} \mathrm{Cl}$ and ethanol treatment as described ${ }^{46}$. The ribosome complexes were assembled on the synthetic 'start-stop' mRNA (5'-GGCAAGGAGGUAAAUAAUGUAAACGAUU-3') as follows: 70S $(1 \mu \mathrm{M})$, initiation factors IF1, IF2 $(2 \mu \mathrm{M}) \overline{\text { and }}$ IF3 $(1.5 \mu \mathrm{M})$, start-stop mRNA $(3 \mu \mathrm{M})$ and $\left.\mathrm{fl}^{3} \mathrm{H}\right]$ Met-tRNA ${ }^{\mathrm{fMet}}(1.5 \mu \mathrm{M})$ were incubated in buffer $\mathrm{A}(30 \mathrm{mM}$ HEPES, pH 7.4, $70 \mathrm{mM} \mathrm{NH}_{4} \mathrm{Cl}, 5 \mathrm{mM} \mathrm{MgCl}_{2}, 30 \mathrm{mM} \mathrm{KCl}$ ) in the presence of GTP $(1 \mathrm{mM})$ for $30 \mathrm{~min}$ at $37^{\circ} \mathrm{C}$ and purified through sucrose cushion as described ${ }^{23}$. The
}

ribosome pellets were resuspended in buffer $\mathrm{A}$, flash frozen in liquid nitrogen, and stored at $-80^{\circ} \mathrm{C}$

E. coli strain and growth conditions. E. coli BL21 strain was used for the expression of RF1-GAQ and RF3. Cells were grown in LB medium supplemented with the required antibiotic, and expression was induced by addition of $0.5 \mathrm{mM}$ IPTG.

Purification of peptide chain release factors. RF1, RF1-GAQ (RF1-G234A), RF3 and the single-cysteine RF1-GAQ were expressed and purified by affinity chromatography as described 22,26 . RF1-GAQ was labelled with Qsy926.

Cryo-grid preparation for the 70S-tRNA-RF1-RF3 complex. All following steps were performed in buffer A (30 mM HEPES, pH 7.4, $70 \mathrm{mM} \mathrm{NH}_{4} \mathrm{Cl}, 5 \mathrm{mM} \mathrm{MgCl}$ $30 \mathrm{mM} \mathrm{KCl}$ ). For grid preparation, $5 \mathrm{OD} \mathrm{A}_{260}$ per $\mathrm{ml}$ of ribosomes were used. RF3 was initially incubated with $1 \mathrm{mM}$ of GDPCP at $37^{\circ} \mathrm{C}$ for $15 \mathrm{~min}$. Subsequently, the ribosome-tRNA complexes were incubated with a $2.5 \times$ excess of the RF1-GAQ mutant, which is 3300 -fold slower than the wild-type $\mathrm{RF} 1^{7,9}$, and with or without $50 \mu \mathrm{M}$ API (NovoPro Biosciences Inc.) for $1 \mathrm{~min}$ at room temperature. Afterwards a $7.5 \times$ excess of RF3-GDPCP over 70S ribosomes was added to the RF1-GAQ containing ribosome complexes and kept on ice for $<5 \mathrm{~min}$ before cryo-grid preparation. All samples were applied to $2 \mathrm{~nm}$ precoated Quantifoil R3/3 holey carbon supported grids and vitrified using a Vitrobot Mark IV (FEI, Netherlands).

\section{Cryo-electron microscopy and single-particle reconstruction. The low-} resolution data collection of the 70S-tRNA-RF1-RF3 complex, which was prepared in the absence of API, was conducted using a Tecnai G2 Spirit (FEI) transmission electron microscope (TEM) equipped with a TemCam-F816 camera (TVIPS) at $120 \mathrm{kV}$ using a pixel size of $2.85 \AA$. The high-resolution data collection of the 70S-tRNA-RF1-RF3 complex, which was prepared in the presence of API, was performed using an FEI Titan Krios TEM equipped with a Falcon II (FEI) direct electron detector at $300 \mathrm{kV}$ using a pixel size of $1.061 \AA$ and an under-defocus range of -0.8 to $-1.6 \mu \mathrm{m}$ resulting in a total number of 5670 micrographs. Each micrograph was recorded as a series of 16 frames $\left(2.7 \mathrm{e}^{-}\right.$per $\AA^{2}$ pre-exposure; $2.7 \mathrm{e}^{-}$per $\AA^{2}$ dose per frame). All frames (accumulated dose of $45.9 \mathrm{e}^{-}$per $\AA^{2}$ ) were aligned using the Unblur software ${ }^{63}$, and power spectra, defocus values, astigmatism and estimation of micrograph resolution were determined by GCTF 64 Micrographs showing Thon rings beyond 4.0 Å resolution were further used. Automatic particle picking was performed using Gautomatch (http://www.mrclmb.cam.ac.uk/kzhang/), and single particles were processed using the Relion2.1 software package ${ }^{31}$. Initial 2D classification/alignment was performed with 703,379 particles. Subsequently, promising 2D classes with a total number of 525,595 ribosomal particles were selected and subjected to $3 \mathrm{D}$ refinement using an E. coli-70S ribosome as a reference structure. Initial alignment and subsequent 3D classification was performed using three times decimated data. The initially refined particles were $3 \mathrm{D}$ classified into 8 classes. Class $1-4$ were refined again and subjected to another 3D classification. Sorting of class $1-4$ resulted in $4,4,3$ or 3 additional subclasses, respectively. The most stable sub-class of each $3 \mathrm{D}$ classification was then $3 \mathrm{D}$-refined. To further increase the resolution of RF3, we applied a focussed mask on RF3. For 3D classification, the same reference was used as for the $3 \mathrm{D}$ refinement. The maximum resolution was observed for state I and state II, extending to $<3.9 \AA$ (FSC ${ }_{0.143}$ ) (Supplementary Fig. 1). The local resolution of the final maps was computed using ResMap ${ }^{65}$ (Supplementary Fig. 2). The final maps were sharpened by dividing the maps by the modulation transfer function of the detector and by applying an automatically determined negative $B$ factor to the maps using Relion $2.1^{31}$. For model building the final maps were locally filtered using the SPHIRE cryo-EM software suite ${ }^{66}$. Resolution was estimated using the "gold standard" criterion (FSC $=0.143)^{67,68}$.

Molecular modelling and map-docking procedures. The molecular models for the ribosome were based on the E. coli-70S-EF-Tu structure (PDB: 5AFI) ${ }^{69}$. The models of RF3 and GDPCP are based on the structure of E. coli RF3-GDPNP bound to Thermus thermophilus 70S (PDB: 4V85) ${ }^{29}$. The structure of RF1-GAQ and API is based on the recently published E. coli-70S-API-RF1 structure (PDB: $5 \mathrm{O} 2 \mathrm{R})^{26}$. The $\mathrm{tRNA}^{\mathrm{fMet}}$ in the classical state is derived from an E. coli $70 \mathrm{~S}$ initiation complex containing the ribosomal rescue factor ArfA (PDB: 5U9F) ${ }^{70}$ The tRNA ${ }^{\text {fMet }}$ in the P/E hybrid state is based on the hybrid state tRNA ${ }^{\text {fMet }}$ from the T. thermophilus RF3-GDPCP-70S structure (PDB: 4V8O) ${ }^{28}$. The rRNA domains and proteins were rigid-body fitted into the respective EM-map using Chimera $^{71}$. The models were manually adjusted and refined using $\operatorname{Coot}^{72}$. Due to the lack of density, domain I of RF1-GAQ was not modelled for states I-II and IV, whereas it was possible to generate a poly-Ala model of domain I of RF1-GAQ for state III. The complete atomic model of the respective complexes were refined into the locally filtered maps using phenix.real_space_refine with secondary structure restraints calculated by PHENIX $1.13^{73}$. Cross-validation against overfitting was performed as described elsewhere ${ }^{74}$. The statistics of the refined model were obtained using MolProbity ${ }^{75}$ (Table 1). 
Calculation of rotation angles. Rotation angles were calculated using PyMol Molecular Graphics Systems (Version 1.8 Schrödinger) (Supplementary Table 1). The body/platform (including h44) rotation was calculated relatively to the 50S ribosomal subunit. Accordingly, for body/platform rotations the molecular models were aligned based on the $23 \mathrm{~S}$ rRNA using state I (this study) as reference. The head swivel was calculated relatively to the $30 \mathrm{~S}$ body/platform. In order to get comparable values for head swivelling, the compared molecular models were aligned based on the 16S rRNA of the body/platform using state I (this study) as reference.

RMSD and vector calculations. Root mean square deviation (RMSD) values were calculated between related alpha-carbon (protein) or phosphate atoms (rRNA) using PyMol Molecular Graphics Systems (Version 1.8 Schrödinger). The reported RF1-API-70S structure ${ }^{26}$ was used as reference. All compared models were aligned to the $50 \mathrm{~S}$ subunit of state I (this study). The $30 \mathrm{~S}$ protein or rRNA residues of the reference structure were coloured according to the determined RMSD of each atom. Vectors were calculated between shifted alpha-carbon and phosphate atoms using the same reference ${ }^{26}$. The vectors were coloured based on their length (distance between atoms).

Peptide hydrolysis. Ribosome termination complexes $(10 \mathrm{nM})$ prepared with wild type or $\Delta \mathrm{bL} 12$ ribosomes were mixed with increasing concentrations of RF1 for $10 \mathrm{~s}$ at $37^{\circ} \mathrm{C}$ in buffer A. Reactions were quenched with a solution containing TCA (10\%) and ethanol (50\%). After centrifugation $(30 \mathrm{~min}, 16,000 \times g)$ the amount of released $\mathrm{f}\left[{ }^{3} \mathrm{H}\right]$ Met in the supernatant was quantified by radioactive counting.

Rapid kinetics. Rapid kinetic experiments were performed on an SX-20MV stopped-flow apparatus (Applied Photophysics, Leatherhead, UK), by rapidly mixing equal volumes $(60 \mu \mathrm{l})$ of reactants at $37^{\circ} \mathrm{C}$ in buffer A. Binding of RF1GAQ was monitored by mixing termination complex labelled with fluorescein $(50 \mathrm{nM})$ with RF1-GAQ ${ }_{\mathrm{QSY}}(300 \mathrm{nM})$. Fluorescein was excited at $470 \mathrm{~nm}$ and monitored after passing a KV500 filter (Schott, Mainz, Germany).

Figure preparation. All figures showing electron densities and atomic models were generated using UCSF Chimera ${ }^{71}$ and PyMol Molecular Graphics Systems (Version 1.8 Schrödinger).

Data availability. The data that support the findings of this study are available from the corresponding author upon request. The atomic coordinates and/or the associated maps have been deposited in the PDB and/or EMDB with the accession codes EMD: 0076/PDB: 6GWT (State I), EMD: 0080/PDB: 6GXM (State II), EMD: 0081/PDB: 6GXN (State III), EMD: 0082/PDB: 6GXO (State IV) and EMD: 0083/ PDB: 6GXP (RF3-70S).

Received: 13 April 2018 Accepted: 6 July 2018

Published online: 03 August 2018

\section{References}

1. Capecchi, M. R. Polypeptide chain termination in vitro: isolation of a release factor. Proc. Natl Acad. Sci. USA 58, 1144-1151 (1967).

2. Scolnick, E., Tompkins, R., Caskey, T. \& Nirenberg, M. Release factors differing in specificity for terminator codons. Proc. Natl Acad. Sci. USA 61 , 768-774 (1968).

3. Nakamura, Y., Ito, K. \& Isaksson, L. A. Emerging understanding of translation termination. Cell 87, 147-150 (1996).

4. Dunkle, J. A. \& Cate, J. H. Ribosome structure and dynamics during translocation and termination. Annu Rev. Biophys. 39, 227-244 (2010).

5. Frolova, L. et al. Mutations in the highly conserved GGQ motif of class 1 polypeptide release factors abolish the ability of human eRF1 to trigger peptidyl-tRNA hydrolysis. RNA 5, 1014-1020 (1999).

6. Seit-Nebi, A., Frolova, L., Justesen, J. \& Kisselev, L. Class-1 translation termination factors: invariant GGQ minidomain is essential for release activity and ribosome binding but not for stop codon recognition. Nucleic Acids Res. 29, 3982-3987 (2001).

7. Zavialov, A. V., Mora, L., Buckingham, R. H. \& Ehrenberg, M. Release of peptide promoted by the GGQ motif of class 1 release factors regulates the GTPase activity of RF3. Mol. Cell 10, 789-798 (2002).

8. Mora, L. et al. The essential role of the invariant GGQ motif in the function and stability in vivo of bacterial release factors RF1 and RF2. Mol. Microbiol. 47, 267-275 (2003).

9. Shaw, J. J. \& Green, R. Two distinct components of release factor function uncovered by nucleophile partitioning analysis. Mol. Cell 28, 458-467 (2007).
10. Laurberg, M. et al. Structural basis for translation termination on the $70 \mathrm{~S}$ ribosome. Nature 454, 852-857 (2008).

11. Korostelev, A. et al. Crystal structure of a translation termination complex formed with release factor RF2. Proc. Natl Acad. Sci. USA 105, 19684-19689 (2008).

12. Weixlbaumer, A. et al. Insights into translational termination from the structure of RF2 bound to the ribosome. Science 322, 953-956 (2008).

13. Jin, H., Kelley, A. C., Loakes, D. \& Ramakrishnan, V. Structure of the $70 \mathrm{~S}$ ribosome bound to release factor 2 and a substrate analog provides insights into catalysis of peptide release. Proc. Natl Acad. Sci. USA 107, 8593-8598 (2010).

14. Zhou, J., Korostelev, A., Lancaster, L. \& Noller, H. F. Crystal structures of 70S ribosomes bound to release factors RF1, RF2 and RF3. Curr. Opin. Struct. Biol. 22, 733-742 (2012).

15. Goldstein, J. L. \& Caskey, C. T. Peptide chain termination: effect of protein S on ribosomal binding of release factors. Proc. Natl Acad. Sci. USA 67, 537-543 (1970).

16. Freistroffer, D. V., Pavlov, M. Y., MacDougall, J., Buckingham, R. H. \& Ehrenberg, M. Release factor RF3 in E. coli accelerates the dissociation of release factors RF1 and RF2 from the ribosome in a GTP-dependent manner. EMBO J. 16, 4126-4133 (1997).

17. Gao, H. et al. RF3 induces ribosomal conformational changes responsible for dissociation of class I release factors. Cell 129, 929-941 (2007).

18. Kihira, K. et al. Crystal structure analysis of the translation factor RF3 (release factor 3). FEBS Lett. 586, 3705-3709 (2012).

19. Pel, H., Moffat, J., Ito, K., Nakamura, Y. \& Tate, W. Escherichia coli relaease fator 3: Resolving the paradox of a typical $G$ protein structure and atypical function with guanine nucleotides. RNA 4, 47-54 (1998).

20. Zavialov, A. V., Buckingham, R. H. \& Ehrenberg, M. A posttermination ribosomal complex is the guanine nucleotide exchange factor for peptide release factor RF3. Cell 107, 115-124 (2001).

21. Koutmou, K. S., McDonald, M. E., Brunelle, J. L. \& Green, R. RF3:GTP promotes rapid dissociation of the class 1 termination factor. RNA $\mathbf{2 0}$, 609-620 (2014).

22. Adio, $\mathrm{S}$. et al. Dynamics of ribosomes and release factors during translation termination in E. coli. eLife 7, e34252 (2018).

23. Peske, F., Kuhlenkoetter, S., Rodnina, M. V. \& Wintermeyer, W. Timing of GTP binding and hydrolysis by translation termination factor RF3. Nucleic Acids Res. 42, 1812-1820 (2014).

24. Ermolenko, D. N. et al. Observation of intersubunit movement of the ribosome in solution using FRET. J. Mol. Biol. 370, 530-540 (2007).

25. Sternberg, S. H., Fei, J., Prywes, N., McGrath, K. A. \& Gonzalez, R. L. Jr. Translation factors direct intrinsic ribosome dynamics during translation termination and ribosome recycling. Nat. Struct. Mol. Biol. 16, 861-868 (2009).

26. Florin, T. et al. An antimicrobial peptide that inhibits translation by trapping release factors on the ribosome. Nat. Struct. Mol. Biol. 24, 752-757 (2017).

27. Klaholz, B., Myasnikov, A. \& Van Heel, M. Visualization of release factor 3 on the ribosome during termination of protein synthesis. Nature 427, 862-865 (2004).

28. Jin, H., Kelley, A. C. \& Ramakrishnan, V. Crystal structure of the hybrid state of ribosome in complex with the guanosine triphosphatase release factor 3 . Proc. Natl Acad. Sci. USA 108, 15798-15803 (2011).

29. Zhou, J., Lancaster, L., Trakhanov, S. \& Noller, H. F. Crystal structure of release factor RF3 trapped in the GTP state on a rotated conformation of the ribosome. RNA 18, 230-240 (2012).

30. Pallesen, J. et al. Cryo-EM visualization of the ribosome in termination complex with apo-RF3 and RF1. eLife 2, e00411 (2013).

31. Kimanius, D., Forsberg, B. O., Scheres, S. H. \& Lindahl, E. Accelerated cryoEM structure determination with parallelisation using GPUs in RELION-2. eLife 5, e18722 (2016).

32. Fischer, N. et al. The pathway to GTPase activation of elongation factor SelB on the ribosome. Nature 540, 80-85 (2016).

33. Fischer, N., Konevega, A. L., Wintermeyer, W., Rodnina, M. V. \& Stark, H. Ribosome dynamics and tRNA movement by time-resolved electron cryomicroscopy. Nature 466, 329-333 (2010).

34. Zhang, W., Dunkle, J. A. \& Cate, J. H. Structures of the ribosome in intermediate states of ratcheting. Science 325, 1014-1017 (2009).

35. Julian, P. et al. Structure of ratcheted ribosomes with tRNAs in hybrid states. Proc. Natl Acad. Sci. USA 105, 16924-16927 (2008).

36. Agirrezabala, X. et al. Structural characterization of mRNA-tRNA translocation intermediates. Proc. Natl Acad. Sci. USA 109, 6094-6099 (2012).

37. Brilot, A. F., Korostelev, A. A., Ermolenko, D. N. \& Grigorieff, N. Structure of the ribosome with elongation factor $\mathrm{G}$ trapped in the pretranslocation state. Proc. Natl Acad. Sci. USA 110, 20994-20999 (2013). 
38. Maracci, C. \& Rodnina, M. V. Review: translational GTPases. Biopolymers 105, 463-475 (2016).

39. Myasnikov, A. et al. Conformational transition of initiation factor 2 from the GTP- to GDP-bound state visualized on the ribosome. Nat. Struct. Mol. Biol. 12, 1145-1149 (2005).

40. Sprink, T. et al. Structures of ribosome-bound initiation factor 2 reveal the mechanism of subunit association. Sci. Adv. 2, e1501502 (2016).

41. Behrmann, E. et al. Structural snapshots of actively translating human ribosomes. Cell 161, 845-857 (2015).

42. Shao, $\mathrm{S}$. et al. Decoding mammalian ribosome-mRNA states by translational GTPase complexes. Cell 167, 1229-1240 e15 (2016).

43. Loveland, A. B., Demo, G., Grigorieff, N. \& Korostelev, A. A. Ensemble cryo-EM elucidates the mechanism of translation fidelity. Nature 546, 113-117 (2017).

44. Carlson, M. A. et al. Ribosomal protein L7/L12 is required for GTPase translation factors EF-G, RF3, and IF2 to bind in their GTP state to 70S ribosomes. FEBS J. 284, 1631-1643 (2017).

45. Ge, X., Mandava, C. S., Lind, C., Aqvist, J. \& Sanyal, S. Complementary chargebased interaction between the ribosomal-stalk protein L7/12 and IF2 is the key to rapid subunit association. Proc. Natl Acad. Sci. USA 115, 4649-4654 (2018).

46. Mohr, D., Wintermeyer, W. \& Rodnina, M. V. GTPase activation of elongation factors $\mathrm{Tu}$ and $\mathrm{G}$ on the ribosome. Biochemistry 41, 12520-12528 (2002).

47. Diaconu, M. et al. Structural basis for the function of the ribosomal L7/12 stalk in factor binding and GTPase activation. Cell 121, 991-1004 (2005).

48. Helgstrand, M. et al. The ribosomal stalk binds to translation factors IF2, EF$\mathrm{Tu}, \mathrm{EF}-\mathrm{G}$ and RF3 via a conserved region of the L12 C-terminal domain. J. Mol. Biol. 365, 468-479 (2007).

49. Savelsbergh, A., Mohr, D., Kothe, U., Wintermeyer, W. \& Rodnina, M. V. Control of phosphate release from elongation factor $\mathrm{G}$ by ribosomal protein L7/12. EMBO J. 24, 4316-4323 (2005).

50. Kothe, U., Wieden, H. J., Mohr, D. \& Rodnina, M. V. Interaction of helix D of elongation factor Tu with helices 4 and 5 of protein L7/12 on the ribosome. J. Mol. Biol. 336, 1011-1021 (2004).

51. Mora, L., Zavialov, A., Ehrenberg, M. \& Buckingham, R. H. Stop codon recognition and interactions with peptide release factor RF3 of truncated and chimeric RF1 and RF2 from Escherichia coli. Mol. Microbiol. 50, 1467-1476 (2003).

52. Klaholz, B. P. et al. Structure of the Escherichia coli ribosomal termination complex with release factor 2. Nature 421, 90-94 (2003).

53. Rawat, U. B. et al. A cryo-electron microscopic study of ribosome-bound termination factor RF2. Nature 421, 87-90 (2003).

54. Shi, X. \& Joseph, S. Mechanism of translation termination: RF1 dissociation follows dissociation of RF3 from the ribosome. Biochemistry 55, 6344-6354 (2016).

55. Dunkle, J. A. et al. Structures of the bacterial ribosome in classical and hybrid states of tRNA binding. Science 332, 981-984 (2011).

56. Barat, C. et al. Progression of the ribosome recycling factor through the ribosome dissociates the two ribosomal subunits. Mol. Cell 27, 250-261 (2007).

57. Seo, H. et al. Kinetics and thermodynamics of RRF, EF-G, and thiostrepton interaction on the Escherichia coli ribosome. Biochemistry 43, 12728-12740 (2004).

58. Borg, A., Pavlov, M. \& Ehrenberg, M. Complete kinetic mechanism for recycling of the bacterial ribosome. RNA 22, 10-21 (2016).

59. Savelsbergh, A., Rodnina, M. V. \& Wintermeyer, W. Distinct functions of elongation factor $\mathrm{G}$ in ribosome recycling and translocation. RNA 15, 772-780 (2009).

60. Peske, F., Rodnina, M. \& Wintermeyer, W. Sequence of steps in ribosome recycling as defined by kinetic analysis. Mol. Cell 18, 403-412 (2005).

61. Milon, P. et al. Transient kinetics, fluorescence, and FRET in studies of initiation of translation in bacteria. Methods Enzymol. 430, 1-30 (2007).

62. Rodnina, M. V. \& Wintermeyer, W. GTP consumption of elongation factor Tu during translation of heteropolymeric mRNAs. Proc. Natl Acad. Sci. USA 92, 1945-1949 (1995).

63. Grant, T. \& Grigorieff, N. Measuring the optimal exposure for single particle cryo-EM using a 2.6 A reconstruction of rotavirus VP6. eLife 4, e06980 (2015).

64. Zhang, K. Gctf: real-time CTF determination and correction. J. Struct. Biol. 193, 1-12 (2016)

65. Kucukelbir, A., Sigworth, F. J. \& Tagare, H. D. Quantifying the local resolution of cryo-EM density maps. Nat. Methods 11, 63-65 (2014).

66. Moriya, T. et al. High-resolution single particle analysis from electron cryomicroscopy images using SPHIRE. J. Vis. Exp. https://doi.org/10.3791/ 55448 (2017).
67. Rosenthal, P. \& Henderson, R. Optimal determination of particle orientation, absolute hand, and control loss in single particle electron microscopy. J. Mol. Biol. 333, 721-745 (2003).

68. Scheres, S. H. \& Chen, S. Prevention of overfitting in cryo-EM structure determination. Nat. Methods 9, 853-854 (2012).

69. Fischer, N. et al. Structure of the E. coli ribosome-EF-Tu complex at $<3 \mathrm{~A}$ resolution by C-corrected cryo-EM. Nature 520, 567-570 (2015).

70. Demo, G. et al. Mechanism of ribosome rescue by ArfA and RF2. eLife 6, e23687 (2017)

71. Pettersen, E. F. et al. UCSF chimera - a visualization system for exploratory research and analysis. J. Comput. Chem. 25, 1605-1612 (2004).

72. Emsley, P. \& Cowtan, K. Coot: model-building tools for molecular graphics. Acta Crystallogr. Sect. D. - Biol. Crystallogr. 60, 2126-2132 (2004).

73. Adams, P. D. et al. PHENIX: a comprehensive Python-based system for macromolecular structure solution. Acta Crystallogr. D Biol. Crystallogr. 66, 213-221 (2010).

74. Brown, A. et al. Tools for macromolecular model building and refinement into electron cryo-microscopy reconstructions. Acta Crystallogr. D Biol. Crystallogr. 71, 136-153 (2015).

75. Chen, V. B. et al. MolProbity: all-atom structure validation for macromolecular crystallography. Acta Crystallogr. D Biol. Crystallogr. 66, 12-21 (2010).

\section{Acknowledgements}

This work has been supported by iNEXT, project number 2992, funded by the Horizon 2020 programme of the European Union. This article reflects only the author's view and the European Commission is not responsible for any use that may be made of the information it contains. CIISB research infrastructure project LM2015043 funded by MEYS CR is gratefully acknowledged for the financial support of the measurements at the CF Cryo-electron Microscopy and Tomography CEITEC MU. This research was supported by grants of the Forschergruppe FOR1805 (to D.N.W. and M.V.R) and WI3285/6-1 (to D.N.W.) from the Deutsche Forschungsgemeinschaft (DFG).

\section{Author contributions}

D.N.W and M.V.R. designed the study, M.G. and C.M. prepared the cryo-EM sample, M.P. collected the cryo-EM data, M.G. processed the cryo-EM data, M.G. and P.H. built and refined the molecular models, M.G., P.H. and D.N.W. analyzed the cryo-EM data. C. M. performed bL12 experiments. M.G. prepared the figures. M.G. and D.N.W. wrote the paper with help from C.M. and M.V.R.

\section{Additional information}

Supplementary Information accompanies this paper at https://doi.org/10.1038/s41467018-05465-1.

Competing interests: The authors declare no competing interests.

Reprints and permission information is available online at http://npg.nature.com/ reprintsandpermissions/

Publisher's note: Springer Nature remains neutral with regard to jurisdictional claims in published maps and institutional affiliations.

Open Access This article is licensed under a Creative Commons Attribution 4.0 International License, which permits use, sharing, adaptation, distribution and reproduction in any medium or format, as long as you give appropriate credit to the original author(s) and the source, provide a link to the Creative Commons license, and indicate if changes were made. The images or other third party material in this article are included in the article's Creative Commons license, unless indicated otherwise in a credit line to the material. If material is not included in the article's Creative Commons license and your intended use is not permitted by statutory regulation or exceeds the permitted use, you will need to obtain permission directly from the copyright holder. To view a copy of this license, visit http://creativecommons.org/ licenses/by/4.0/.

(c) The Author(s) 2018 